\title{
Taking Mental Health \& Well-Being to the Streets: An Exploratory Evaluation of In-Vehicle Interventions in the Wild
}

\author{
Kevin Koch* \\ kevin.koch@unisg.ch \\ University of St. Gallen \\ St. Gallen, Switzerland
}

\author{
Thomas Berger \\ thomas.berger@psy.unibe.ch \\ University of Bern \\ Bern, Switzerland
}

\author{
Verena Tiefenbeck \\ University of Erlangen-Nuremberg \\ Nuremberg, Germany \\ ETH Zürich \\ Zürich, Switzerland \\ verena.tiefenbeck@fau.de \\ Elgar Fleisch \\ ETH Zürich \\ Zürich, Switzerland \\ University of St. Gallen \\ St. Gallen, Switzerland \\ efleisch@ethz.ch
}

\author{
Shu Liu \\ liush@ethz.ch \\ ETH Zürich \\ Zürich, Switzerland
}

Felix Wortmann

felix.wortmann@unisg.ch

University of St. Gallen

St. Gallen, Switzerland

\begin{abstract}
The increasing number of mental disorders worldwide calls for novel types of prevention measures. Given the number of commuters who spend a substantial amount of time on the road, the car offers an opportune environment. This paper presents the first invehicle intervention study affecting mental health and well-being on public roads. We designed and implemented two in-vehicle interventions based on proven psychotherapy interventions. Whereas the first intervention uses mindfulness exercises while driving, the second intervention induces positive emotions through music. Ten ordinary and healthy commuters completed 313 of these interventions on their daily drives over two months. We collected drivers' immediate and post-driving feedback for each intervention and conducted interviews with the drivers after the end of the study. The results show that both interventions have improved drivers' well-being. While the participants rated the music intervention very positively, the reception of the mindfulness intervention was more ambivalent.
\end{abstract}

\section{CCS CONCEPTS}

- Human-centered computing $\rightarrow$ Empirical studies in HCI; Ubiquitous and mobile computing systems and tools; • Applied computing $\rightarrow$ Consumer health.

\footnotetext{
${ }^{*}$ Corresponding author
}

Permission to make digital or hard copies of all or part of this work for personal or classroom use is granted without fee provided that copies are not made or distributed for profit or commercial advantage and that copies bear this notice and the full citation on the first page. Copyrights for components of this work owned by others than the author(s) must be honored. Abstracting with credit is permitted. To copy otherwise, or republish, to post on servers or to redistribute to lists, requires prior specific permission and/or a fee. Request permissions from permissions@acm.org.

CHI '21, May 8-13, 2021, Yokohama, Japan

(c) 2021 Copyright held by the owner/author(s). Publication rights licensed to ACM. ACM ISBN 978-1-4503-8096-6/21/05 .. \$15.00

https://doi.org/10.1145/3411764.3446865

\section{KEYWORDS}

In-vehicle interventions; Psychology; Field study; Natural driving; Mindfulness; Music; Well-being

ACM Reference Format:

Kevin Koch, Verena Tiefenbeck, Shu Liu, Thomas Berger, Elgar Fleisch, and Felix Wortmann. 2021. Taking Mental Health \& Well-Being to the Streets: An Exploratory Evaluation of In-Vehicle Interventions in the Wild. In CHI Conference on Human Factors in Computing Systems (CHI'21), May 8-13, 2021, Yokohama, Japan. ACM, New York, NY, USA, 15 pages. https: //doi.org/10.1145/3411764.3446865

\section{INTRODUCTION}

In recent years, there has been a massive increase in the number of people affected by mental health issues. Over the last decade, major depressive disorders rapidly increased by $18.4 \%$ to 320 million cases per year worldwide and anxiety disorders by $14.9 \%$ to 264 million cases $[67,73]$. Beyond the loss of quality of life for the individual, the resulting economic burden of these diseases is enormous because society is confronted with productivity losses and high treatment costs [27]. Given that less than half of all mental disorders are detected and treated, the World Health Organization has described the situation as alarming [38]. Hence, prevention of mental illnesses has received increased attention in recent years [22]. The demand for large-scale preventive interventions exceeds the available capacity of existing forms of treatments in psychotherapy, which usually involve personal interaction [27]. Novel interventions, however, ubiquitously carried out via the internet or on mobile devices, may provide supplementary, cost-effective forms of support and offer the advantage of being independent of time and place [22, 49]. Many of them aim to reach the masses to prevent diseases before they arise and before longer-lasting harm materializes, for example by strengthening resilience factors such as well-being [22]. The World Health Organization strongly emphasizes the relevance of prevention. As a matter of fact, they define health not only as the absence of disease, but also as "a state of complete physical, social, 


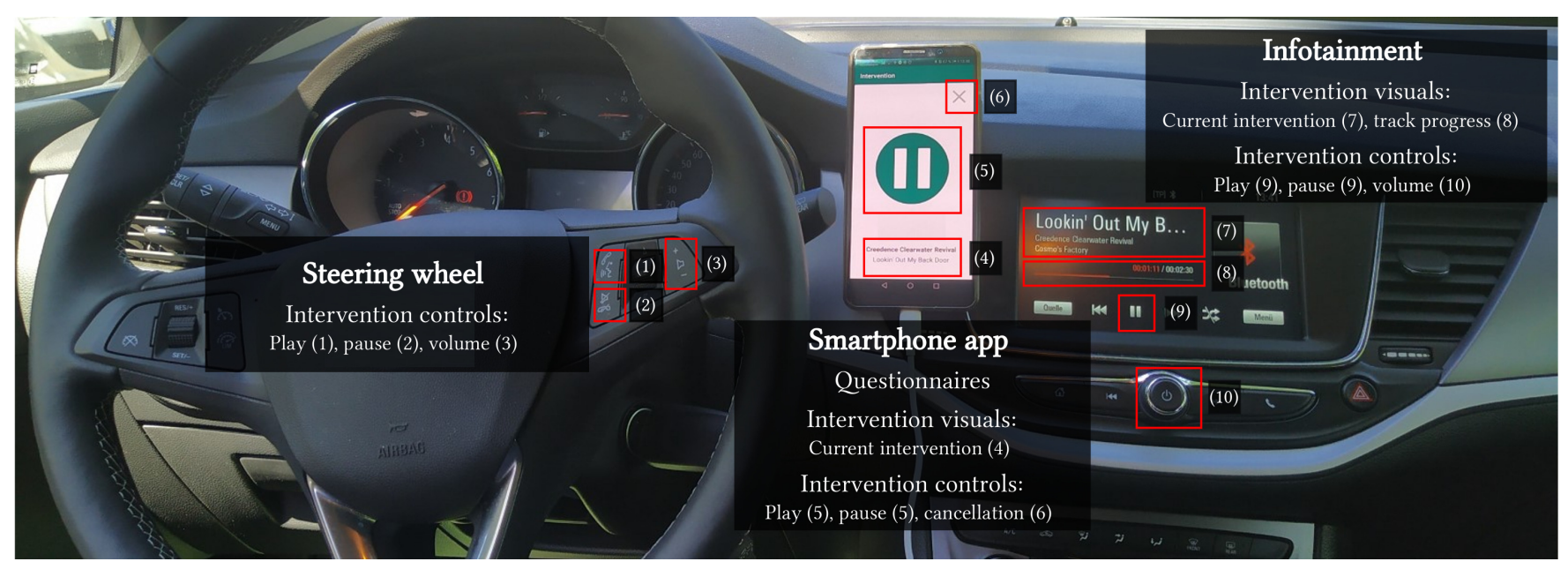

Figure 1: Car interior with the study setup. Left: Buttons on the steering wheel to control the intervention playback. Middle: Smartphone with app to answer the questionnaires and to control the interventions. The snapshot shows the app currently playing an intervention. Right: Car infotainment showing the running intervention.

and emotional well-being " [72]. And indeed, well-being is recognized as a cornerstone to reduce the vulnerability of suffering from mental disorders $[18,24,55]$.

In light of the depicted challenges, psychotherapy extends its reach by bringing interventions into everyday life situations (e.g., by app-based solutions [70]). In this context, existing research has recognized the potential of in-vehicle interventions by exploring novel types of interventions that can be applied in the car without compromising driver safety, such as guided breathing exercises [4] or personalized voice assistants [11]. Commuters in particular spend considerable time in the car that might be used for such interventions. In Germany, for example, approximately $60 \%$ of employees commute by car every day. Among these, over $20 \%$ spend at least one hour per day in their car [62]. Even higher figures apply for the US, where 57 million employees (38\% of the workforce) commute at least one hour per workday [64]. Yet, most existing research in the domain still focuses on laboratory studies or, at most, on real-world settings under highly controlled conditions (i.e., short periods of time or predefined routes). This differs substantially from our study, as we inspect the impact of mental health and well-being interventions in the real world, i.e., on public roads and over a longer period of time.

Today's highly computerized cars enable sophisticated and potentially powerful intervention strategies. However, driver safety is paramount in a real-world environment. Hence, we focus our analysis on fundamental intervention effects and mechanisms, thereby paving the way for more elaborated interaction approaches. More specifically, we developed and implemented two audio-based invehicle interventions: a mindfulness exercise and an emotional music experience. Both intervention types have proven to be highly effective outside of the car; we adapted and implemented them with a multidisciplinary team of computer scientists, psychologists, and driving safety experts for the car. For a period of two months, we have equipped ten participants with cars that were specifically prepared for delivering interventions to analyze the impact of the interventions on public roads. During that time, each driver experienced both types of interventions. More specifically, one intervention was initiated per trip and the type of the intervention was chosen randomly. Upon completion of the intervention, the drivers immediately evaluated the intervention effects on their affective state on the basis of a triggered voice feedback. In addition, the drivers rated their affective state before and after driving. Following previous intervention studies on mental health and well-being, we concentrate on affect, since affective well-being is a specific aspect of well-being closely related to mental illnesses [1, 17, 19].

In short, this paper investigates the impact of two mental health and well-being interventions that had previously proven to be effective outside the car in a longitudinal in-vehicle study on public roads. Thereby, our study addresses the unanswered challenges of whether drivers are able to conduct mental health and wellbeing interventions on open roads, and if so, what their effects are. The contributions of this work are threefold: (1) We transfer and adapt two proven psychotherapeutic interventions for use in the car while driving, (2) we investigate to which extent these in-vehicle interventions for mental health and well-being have an immediate impact on drivers' affect, and (3) we investigate to which extent these in-vehicle interventions have an impact on the drivers' post-driving affect. To the best of our knowledge, we are the first to investigate the effects of in-vehicle interventions in a longitudinal driving study in real-world traffic conditions with everyday commuters.

\section{THEORETICAL BACKGROUND AND RELATED WORK}

In this section, we first outline the term well-being and its relationship to mental health. Next, we inspect existing interventions for the prevention and treatment of mental disorders. Finally, we review existing in-vehicle interventions that are discussed in the literature or are available in today's cars. 


\subsection{Mental Health \& Well-Being}

Empirical evidence strongly suggests that well-being is a crucial factor in increasing resilience against mental illnesses [18, 55]. However, well-being is a rather broad concept that captures physical, mental, and social states [19]. Hence, in the context of mental health, researchers commonly focus on the more specific concept of affective well-being $[1,17]$. Affective well-being refers to individual experiences in everyday life through the frequency and intensity of various emotions such as joy, anger, or affection [17, 68]. It is usually measured by asking people to assess the extent to which they have experienced different affective states over a specific period of time. Common methods of measurement are two- or three-dimensional models, which allow the representation of emotions in a multidimensional space [13].

Empirical research commonly builds upon Russel's circumplex model [54], which measures affective well-being by using two dimensions, i.e., arousal and valence. The former indicates the degree of activation in relation to how tired or energized someone is and the latter measures the degree of happiness. The combination of arousal and valence allows to describe more complex emotions such as excitement (i.e., high arousal and high valence) or being upset (i.e., high arousal and low valence). This rather simple model offers two advantages. First, the measurement is comparable effortless and cross-culturally applicable since standardized measures as the Self-Assessment Manikins exist [10]. Second, arousal and valence can serve as general proxy measures, e.g., for emotions [10], stress [39, 50], and even depression [53]. Therefore, throughout this work, we leverage arousal and valence to understand people's well-being and the impact of our in-vehicle interventions.

\subsection{Interventions for Mental Health \& Well-Being}

The field of psychotherapy deals with the prevention and treatment of mental health disorders. Cognitive Behavioural Therapies (CBT) are perceived to be the current gold standard in psychotherapy and serve as guidelines for many psychotherapeutic methods $[16,30]$. Psychotherapists use CBT either for prevention or treatment to challenge and change people's mindset by making them aware of their emotions and supporting them in their emotion regulation [5]. Most CBT-based treatments consist of several weekly sessions. In each session, psychotherapists work with clients to carry out various exercises or treatments such as psychoeducation, cognitive restructuring, or behavior-change methods [5, 30]. More recently, CBT-based exercises or treatments have been expanded by meditations and an increased focus on emotions [30].

These exercises and treatments have primarily been developed for mentally ill people. However, some of them are designed so that they can also be performed by healthy people for prevention purposes. This applies for example to mindfulness meditation for stress reduction [28] or listening to mood-lifting music [43]. However, these treatments still require intensive individual supervision by psychotherapists [27]. Researchers in the fields of psychotherapy and Human-Computer Interaction (HCI) are already investigating scalable and cost-effective measures for the prevention and treatment of mental disorders, for example with smartphone apps, but the reach of these measures is yet limited $[8,69]$. Popular examples include chatbots that can help with everyday situations such as deand reattachment from work [71], or deliver established psychological treatments for severe conditions (e.g., CBT-based treatment for depressions) $[25,58]$. By deploying in-vehicle interventions, we evaluate a potentially highly scalable approach that a broad population might be able to integrate easily in their daily lives.

\subsection{In-Vehicle Interventions in Literature and Practice}

As of today, affective health in the human-computer interaction community has a strong focus on sensing aspects, whereas intervention studies are still scarce [56]. Although vehicle-related research on this matter is in its infancy, there are already a few intervention studies that focus on well-being of drivers. Previous in-vehicle intervention literature proposes to re-use existing comfort features in the car to improve passengers' well-being. The car, for example, can adapt interior ambient lighting, modify navigation routes (i.e., avoid high traffic routes) or regulate air-conditioning [33].

Recently, speech-based interventions gained major interest in the realm of driving. Specifically, the content and communication between a voice assistant and the driver has been analyzed. A voice assistant can help to cope with frustrating traffic situations by calmly reassessing situations rather than highlighting misbehavior [29]. Furthermore, a personal voice assistant can be adjusted to the driver's personality and the current driving context, which might change the driver's perception of the assistant to be trustworthy and pleasant (e.g., extroverted people tend to prefer a friend-like tone in the assistant's voice) [11]. In addition, novel voice interactions could pave the way for in-vehicle productivity tasks. Recent research examined the creation of PowerPoint slides while driving [46] and the retrieval of document information based on speech alone [63]. Finally, existing literature also investigated the development and testing of in-vehicle voice interactions. Martelaro et al. developed a system that allows researchers to constantly observe a driver using video and audio streams from inside the car, and to communicate with the driver by using a Wizard of Oz-based voice agent [45].

Another related research stream is geared towards guided breathing. Guided breathing exercises were developed and validated in a series of subsequent studies for drivers to better cope with stress while driving $[4,51,52]$. In simulator studies, the researchers first identified possible in-vehicle interventions before designing and validating various breathing exercise patterns [51, 52]. Hereby, a haptic vibrotactile seat cover guided the breathing of drivers to reduce their stress. These studies demonstrate the positive effects of these intervention on physiological and psychological stress in a real car on a dedicated test track [4]. Other studies assessed rather subtle well-being interventions for the car based on scents. In a set of laboratory studies, researchers identified that pleasant scents can calm down drivers [21] and might be effective in helping angry drivers to relax.

The well-being of drivers has also attracted interest in the industry. At least two major car manufacturers introduced the idea of in-vehicle interventions to improve well-being. In 2016, Audi presented the "Audi Fit Driver" concept, which should adapt the vehicle to the driver by interventions based on wearable sensor 
data [2]. Mercedes already launched a very similar solution called "ENERGIZING" in 2018. Vehicles that are shipped with this optional feature package can conduct interventions and e.g., adjust the air-conditioning, start massage seat programs, or change the ambient lighting. These interventions are initialized by a combination of consumer smartwatch data and the car [15]. Unfortunately, these manufacturers have not yet published any results of their interventions.

Summing up, existing research in the field of in-vehicle interventions is gaining momentum, but is still in its infancy. Existing studies have been conducted in highly-controlled environments and most interventions have been tested in driving simulators. The few existing interventions involving actual driving were confined to trips on predefined test tracks or short trips in real traffic. Hence, real-world driving studies covering longer time periods are still missing.

\section{INTERVENTION DESIGN}

For our study design, we drew upon the experience and knowledge of our interdisciplinary research team consisting of computer scientists, psychologists, and driving safety experts. The team iteratively discussed several intervention ideas that originated from established psychotherapeutic exercises. In that process, the team defined arousal and valence of drivers as primary dependent variables to evaluate the positive impact of the interventions. In the end, it was decided to implement two different interventions available to each participant (within-subject), so that enough repetitions by each driver were possible to study longitudinal effects and to assess their experiences for two separate interventions. In order to deliver the interventions during the study, we developed an Android-based smartphone app, which is depicted in Figure 2. In each car, we deployed a smartphone that was running our app right next to the infotainment screen and connected it to the vehicle's multimedia system. This setup is visualized in Figure 1.

\subsection{General Design Principles}

To ensure the safety of the study participants and consider compliance with traffic regulations, the intervention development process followed three main paradigms. First and foremost, we always included a short audio-based warning message at the beginning of each intervention to remind drivers to use the system only in situations (i.e., environment and cognitive state) that do not compromise driver safety. Second, we put drivers in control throughout every intervention. Hence, the participants had to start the intervention by themselves and could stop a running intervention at any moment. Third, we focused on audio-based interventions. Audio-based interventions do not require drivers to read texts or watch videos that could distract their attention from the road. In order to realize a high-quality audio experience, we hired a professional radio announcer to record all audio parts.

\subsection{Mindfulness Intervention}

Recently, mindfulness exercises have attracted attention beyond psychotherapy because empirical evidence suggests that they have beneficial impact on both, mentally ill and healthy people [28, 65]. However, the use of existing mindfulness exercises in the car might (a)

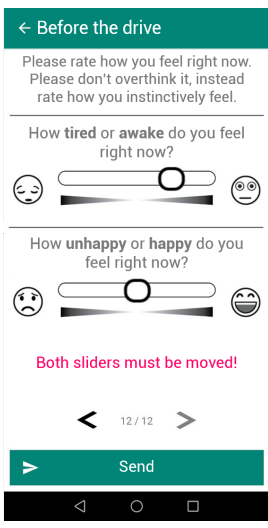

(b)

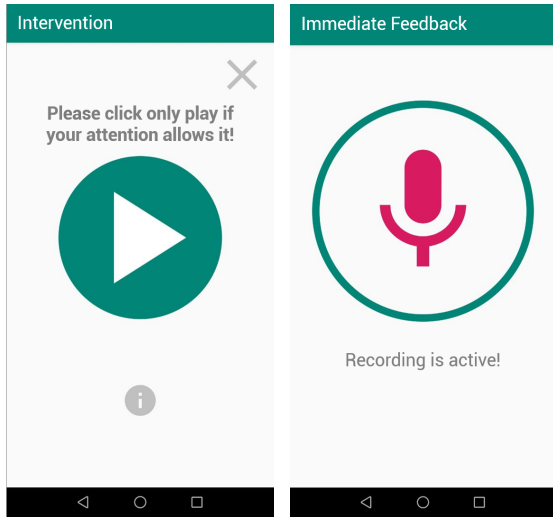

Figure 2: In-vehicle study smartphone app (screens translated). (a) Pre- and post-driving questionnaire (Affective Slider); (b) intervention controls; (c) recording screen for the immediate impact voice questionnaire after an intervention.

be problematic, as most exercises are practiced in forms of a meditation. They build upon closed eyes and silence to experience the two core concepts of mindfulness, i.e., conscious attention on the present and non-judgemental self-perception [3,36]. Obviously, carrying out such meditations while driving might be dangerous. However, there are also passive concepts of mindfulness [31, 37]. These exercises guide listeners to focus on the present and conduct ongoing activities in a more mindful way (e.g., climbing stairs, cooking, or showering). Hence, our first intervention is geared towards driving as a passive mindfulness exercise.

When the mindfulness intervention was played for the first time, it started with instructions on the mindfulness procedure in order to achieve greater acceptance among individuals who were not familiar with the concept. All subsequent interventions directly started with the exercise, which consisted of a sequence of two types of content sections. Environment-centric sections supported the concentration on the surroundings of the car and the current driving task. Self-centric sections helped drivers to reflect their feelings and emotions. Table 1 exemplifies both elements. Each section consisted of three to six text passages that were separated by longer pauses. In line with existing mindfulness exercises, these pauses lasted around 20 seconds and the narration had a slow rhythm of speech. The intervention started off with an environmentcentric section, followed by a self-centric section. Both concepts were repeated once again before a last environment-centric section concluded the exercise. In total, the intervention lasted about 14 minutes. As existing research suggests that mindfulness exercises need to be repeated several times to unfold their impact [36], we have decided to introduce a single exercise that does not change throughout the study.

\subsection{Music Intervention}

The music intervention aims to recall positive memories by playing songs that the participants associate with pleasant moments in 
Table 1: Examples for both mindfulness content blocks (translated).

\begin{tabular}{ll}
\hline Section & Text passage \\
\hline & "What are the other cars nearby doing? \\
Do not judge the driving behaviour of \\
Environment-centric & $\begin{array}{l}\text { the others, just acknowledge it. Do they } \\
\text { drive faster than allowed? Do they seem } \\
\text { stressed or concentrated?" [...] }\end{array}$ \\
& "What do you notice the most right \\
& now? Give this aspect your full atten- \\
& tion and consider it like a scientist. Let \\
& yourself be guided by the sentence: "Ex- \\
citing, what does this do to me?" [...]
\end{tabular}

their lives and that they selected once at the beginning of the study. While music in general has proven to be effective in psychotherapy research [42], more recently, this approach has been justified on the basis of convincing anecdotal evidence in psychotherapeutic practice [6]. The underlying idea is that certain songs help individuals to recall beneficial autobiographical memories that improve valence but also, to a lesser extent, states of arousal [35]. While music itself has effects on affective states (e.g., by speed, volume, rhythm, or other structural elements) [43,59], the music intervention builds in particular on the effect of recalling positive memories. As more than $90 \%$ of car drivers listen to music while driving [61], we conjectured that music is a natural choice for in-vehicle interventions.

Prior to the study, each participant had to create a list of at least ten positive memories. For each memory they had to name a song that they associated with the memory. The result of this process was a personalized playlist with a minimum of ten songs. The average playlist created by the participants comprised 15.6 songs. An excerpt of a participant's playlist is exemplified in Table 2. The music intervention started with a short instruction inviting the driver to enjoy the personal music and to recall past memories. The instruction was general, no explicit links were made to specific memories. After the instruction, the intervention immediately started by playing a random song of the personal playlist. During the playback, participants could pause, cancel or skip songs. The intervention terminated once the total playing time exceeded a minimum of nine minutes and the current song ended. On average, this setup resulted in three songs played.

\subsection{Hypotheses}

Complementary to existing research, we are particularly interested in effects of in-vehicle interventions in real-world driving conditions over a longer period of time. Based on existing literature, we developed six hypotheses that guide our analyses of the immediate and the post-driving intervention effects of our in-vehicle interventions on public roads. Thereby, each hypothesis is examined for each of the two intervention types. To analyze the immediate impact on affect of our in-vehicle interventions, we build upon five hypotheses that assess longitudinal impact as well as the influence of specific variables that have known effects on people's well-being
Table 2: Sample excerpt of a playlist for the music intervention by one participant (translated).

\begin{tabular}{lll}
\hline Memory & Connected song & Artist \\
\hline "Memories of swinging & Total Eclipse of & Bonnie Tyler \\
with my sister in the Heart & \\
grandma's garden" & & \\
"The song of my fiance & Don't Stop Believing & Journey \\
and me" & & \\
& & Die Happy \\
"In a nutshell, my teen & Cry for More & \\
times with my best & & \\
friends" & &
\end{tabular}

(e.g., daytime, day of the week, or the affective state at the beginning of a trip).

$\mathrm{H} 1:$ Both interventions improve the immediate affective state. Depending on their structure and content, mindfulness exercises are able to improve arousal and valence $[9,34]$. Our developed exercise aims to achieve this by helping drivers to focus their attention on the present and by helping them to understand their emotions [34]. Recalling positive emotions on the basis of music, on the other hand, can induce a broad range of emotions [66]. In the realm of our dependent variables, we expect an arousal and valence improvement that is in line with existing research $[35,59]$.

$\mathrm{H} 2$ : Repetitions of the mindfulness intervention increase the immediate impact on affect (H2a), whereas repetitions of the music intervention decrease the immediate impact on affect $(\mathrm{H} 2 b)$. Several repetitions of the mindfulness exercise are needed to unfold their positive effects [9, 34, 37]. By contrast, existing literature suggests that the repeated consumption of (the same, similar) music is associated with a depletion of the stimulus in the long run [14, 57]. In other words, certain songs captivate listeners and delight them until they have heard the song too many times $[44,57]$.

$\mathrm{H} 3$ : Both interventions yield stronger improvements in the evening. People normally exhibit lower affective states in the evening hours, which is known as "daily tiredness" in psychology [23, 47]. Research suggests that such states indicate a situation of "vulnerability" and hence, an intervention completed at that time should prove to be more effective, as there is a larger margin of improvement [49].

$\mathrm{H} 4$ : Both interventions yield stronger improvements on the weekend. Research implies that people tend to have less on their mind on weekends and weekends are vital to recover from the stress of the week [26]. Therefore, we hypothesize that the participants can benefit more from the interventions during the weekend.

$\mathrm{H} 5:$ Both interventions yield stronger improvements if drivers are in a low pre-driving state. If drivers enter the car in low affective states, they are in a state of high "vulnerability" and therefore, an intervention should prove particular effective, as there is 
Table 3: Description of study cohort.

\begin{tabular}{cccccccc}
\hline $\mathbf{n}$ & $\begin{array}{c}\text { Age } \\
\text { (years) }\end{array}$ & Gender & Residence & $\begin{array}{c}\text { Driving per } \\
\text { week } \mathbf{( k m )}\end{array}$ & $\begin{array}{c}\text { Driving days } \\
\text { per week }\end{array}$ & $\begin{array}{c}\text { Distance to } \\
\text { work (km) }\end{array}$ & $\begin{array}{c}\text { Driving } \\
\text { experience } \\
\text { (years) }\end{array}$ \\
\hline 10 & $\mathrm{M}=37.2$ & Female $=4$ & Urban $=3$ & $\mathrm{M}=327.0$ & $\mathrm{M}=6.8$ & $\mathrm{M}=22.6$ & $\mathrm{M}=20.2$ \\
& $\mathrm{SD}=8.4$ & Male $=6$ & Rural $=7$ & $\mathrm{SD}=103.4$ & $\mathrm{SD}=0.4$ & $\mathrm{SD}=7.0$ & $\mathrm{SD}=8.1$ \\
& & & $\min =100$ & $\min =6$ & $\min =14$ & $\min =9$ \\
& & & $\max =500$ & $\max =7$ & $\max =35$ & $\max =38$ \\
\hline
\end{tabular}

more potential for improvement [49]. More specifically, if a driver enters the car in a low arousal (valence) state, interventions should have a distinct impact on arousal (valence).

Regarding longer-lasting effects of our in-vehicle interventions, we concentrate on a single hypothesis to inspect the post-driving impact on affect. Affective changes are known to be transient and less persistent, i.e., people tend to return to their baseline state rather quickly. Against this backdrop, inducing longer-lasting effects would be a major step forward towards sustainable mental health interventions [12]. In essence, we hypothesize that the postdriving affective state is dependent on the immediate improvement resulting from the intervention:

H6: Drivers who perceived a large immediate impact of the intervention, have a higher affective post-driving state. Existing research indicates that interventions with strong immediate effects can have longer-lasting effects on well-being, specifically if the interventions are recurring [48].

\section{FIELD STUDY}

In the following section, we describe our IRB-approved study setup that was deployed in order to evaluate our interventions in the wild over a two-month period (September to November 2019).

\subsection{Participants}

In the recruitment process, we targeted a sample of ten ordinary, healthy daily commuters. We published our call for participation in the social network of a large German company with several offices in urban and rural areas. In total, 54 people responded to this call and filled out a questionnaire about their driving habits. On the basis of their responses, we carefully weighed gender, age, family situation, leisure activities, and driving habits to ensure that our sample comprises a broad spectrum of daily commuters (purposive sampling). Furthermore, the safety of drivers and others on the road was paramount, consequently we only selected participants with long-term driving experience (on average 20.2 years).

All ten participants selected and contacted (four female, six male) agreed to participate in the study. Their age ranged from 26 to 55 years, with a mean of $37.2(\mathrm{SD}=8.4)$ years. Two participants lived alone, eight were in a relationship or married, and three had children. Seven of the participants stated that they like to drive or do not mind driving, at least as long as the road is not busy. The others, however, perceive driving rather as a cumbersome burden.

The participants indicated daily commuting as the main reason for owning a car. In addition, they used their car for leisure activities such as visiting friends or family, regular grocery shopping, or to bring their children to school. All participants lived in or near (i.e., max. 1 hour's drive away from) Stuttgart, Germany. Table 3 provides descriptive statistics of the sample and their commuting patterns. Overall, we consider our sample to comprise a broad spectrum of fairly typical commuters and thus we assume a comparatively high external validity despite the small sample size.

\subsection{Procedure and Data Collection}

Figure 3 illustrates the procedure of a typical trip (including an intervention) in our study. We tested this procedure prior to the study with two volunteers (not involved in the study) during their daily commutes. While their overall feedback was positive, we adjusted minor issues based on their feedback (e.g., shortening the audio announcements or handling incoming voice calls on the private phones of the driver during intervention playback). In the following, the procedure and in particular the data collection will be explained in more detail.

4.2.1 Pre- and Post-Driving Affective State. Before and after each trip, the participants assessed their current affective state by completing the Affective Slider [7] on our smartphone-based study app. The Affective Slider is an empirically validated instrument for measuring arousal and valence, similar to the Self-Assessment Manikin [10]. However, it is adapted to modern user interfaces and devices like smartphones. When using the Affective Slider, the participants rate their perceived state of arousal and valence on two continuous scales from 0 (very low) to 100 (very high). The response before the trip represents the pre-driving or baseline affective state, while the response after the trip reflects the post-driving state. Figure 2(a) visualizes the questionnaire.

4.2.2 Intervention. Directly after the driver completed the predriving affective state self-report, the smartphone app would open the intervention screen. The smartphone app would present a play button (as shown in Figure 2(b)). The drivers only found out which type of intervention (mindfulness or music) would be played after they start the intervention. An intervention starts with a click on play at any time during the trip. To ensure driving safety at all times, drivers could always pause or cancel an ongoing intervention while driving by using the control buttons shown in Figure 1.

4.2.3 Immediate Impact on Affect. To obtain timely feedback on the immediate impact of the interventions, our app started a simple voice-based questionnaire right after the participant had completed an intervention. The study app automatically played the two-item 


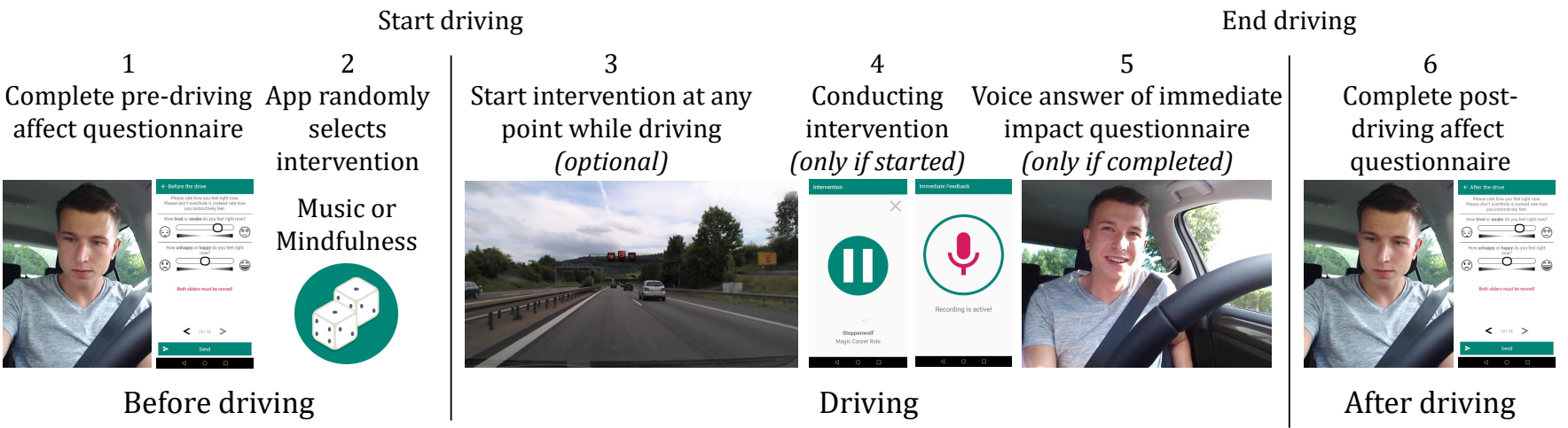

Figure 3: Study procedure for each trip (screens translated).

questionnaire and recorded the participant's answers. The two questions are geared at arousal and valence based on the Client Oriented Scale of Improvement (COSI) [20]. More specifically, our app asked the following two questions (translated from German): "After the intervention, do you feel more aroused (respectively happier) than before?" The participants could indicate their answers on a Likert scale from 0 ("no improvement at all") to 4 ("very strong improvement"). Before the start of the study, the participants received a briefing on the meaning and procedure of this questionnaire in order to guarantee a safe questioning and to clarify possible ambiguities. In summary, the questionnaire allowed us to quantify the immediate impact on affect for the participants after an intervention. The recording is depicted in Figure 2(c).

\section{ANALYSIS AND RESULTS}

In this section, we present the analysis of our hypotheses and a brief summary of the participants' experiences with the interventions.

\subsection{Quantitative Analysis and Results}

For the quantitative analysis, we first provide a short description of the methods used and data collected. We then analyze the mindfulness intervention before focusing on the music intervention. For each intervention, we initially conduct a descriptive data analysis a) to explore the characteristics of the data and b) to identify high-level longitudinal trends that affect all participants alike. More specifically, the analysis assesses the immediate intervention impact over the study duration, the days of the week, and the times of the day. To rigorously disentangle within and in-between subject effects, our consecutive analysis comprises several mixed-effects models that allow us to investigate our hypotheses in detail. We include the drivers as random effects and the remaining independent variables as fixed effects in our models. In calculating the p-value of the fixed-effects, we apply Satterthwaite's degrees of freedom method [40].

To examine $H 1$, we analyze the immediate impact of the intervention on arousal and valence. These two dependent variables, together with our two interventions (i.e., mindfulness and music), result in four basic models. Next, we extend these basic models by gradually adding independent variables based on our subsequent hypotheses. Apart from the intervention type, the independent

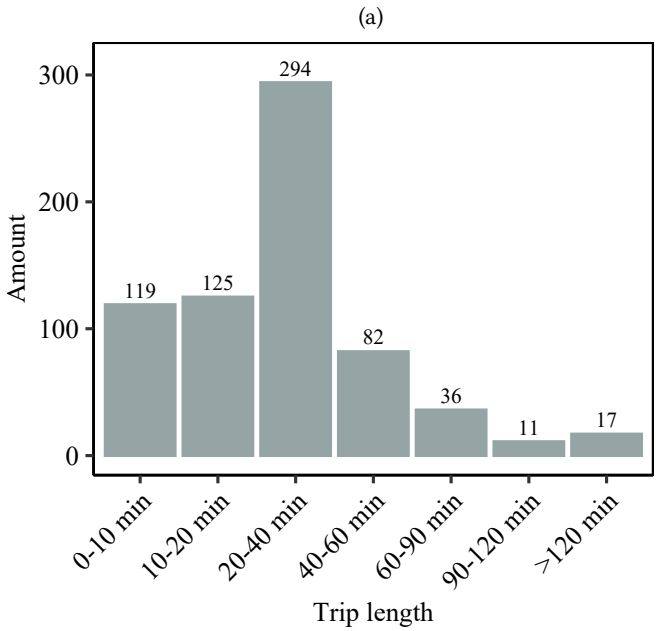

(b)

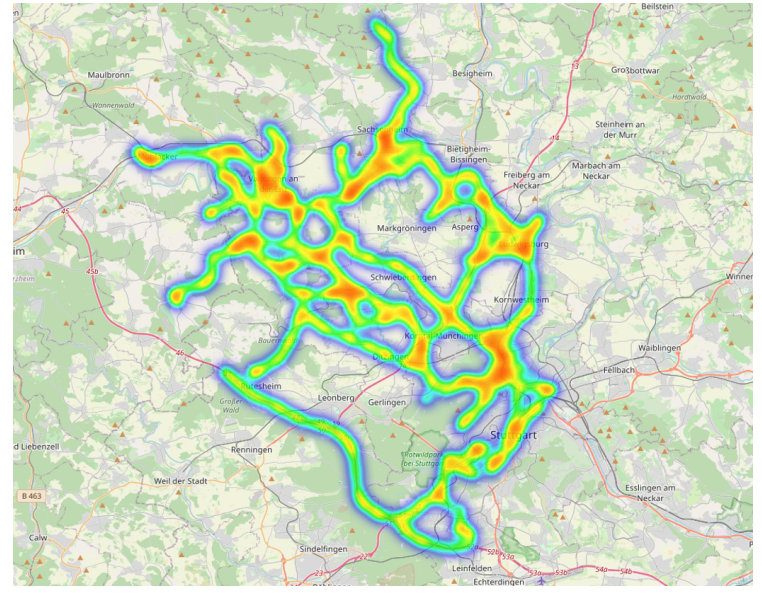

Figure 4: Trip characteristics. (a) Histogram of trips by trip length intervals, aggregated over all participants; (b) GPS heatmap visualizing the geographic driving area of a typical study participant. 
(a)
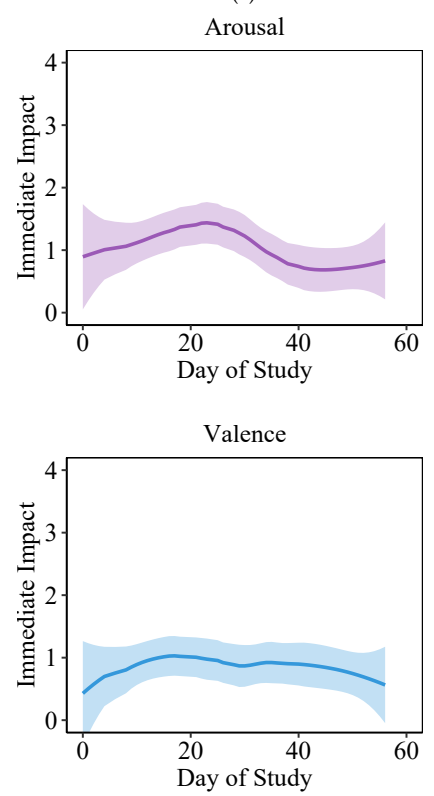

(b)
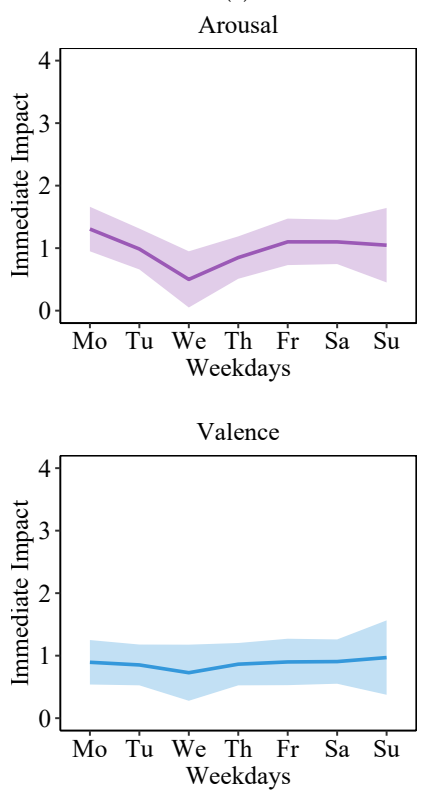

(c)
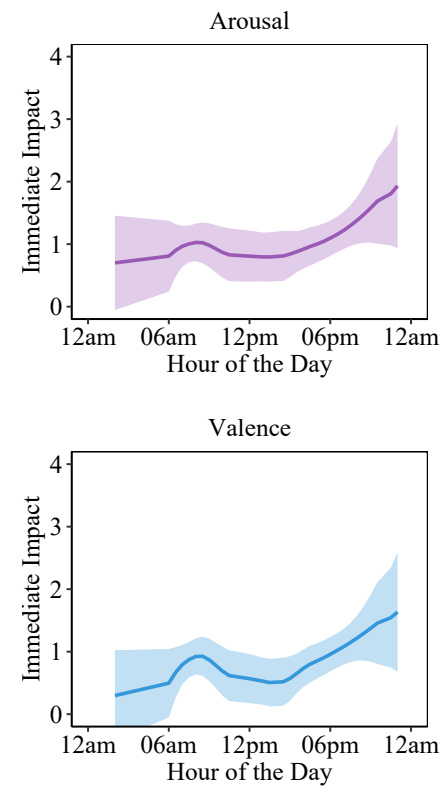

Figure 5: Immediate impact of the mindfulness intervention on arousal (upper row) and valence (lower row). The graphs show the loess-smoothed mean value and the 5 to 95 percentiles for $\mathbf{n}=138$ completed mindfulness interventions. (a) shows the effects over the study duration; (b) shows the effects over the weekdays; and, (c) shows the effects over daytime.

variables include the number of interventions (repetitions) normalized by the natural logarithm function ${ }^{1}$ (to evaluate $H 2$ ); evening (8 pm to midnight) or before (H3); workday or weekend (H4); and a driver's baseline state before driving (H5). Therefore, for each basic model, we have calculated five specifications. By gradually increasing the complexity of the models we follow a simple procedure to evaluate the robustness and stability of the effects. Finally, we evaluate whether the immediate effect of the intervention has an impact on the post-driving state (H6). To that end, we calculate additional mixed-effects models that investigate if the post-driving follows the rationale of "pre-driving state + immediate intervention improvement = post-driving state".

5.1.1 Descriptive Results. During the two months of the study, the drivers had 684 trips with interventions on which they started 460 interventions. Ultimately, the participants successfully completed 313 of these interventions resulting in an average of 31.3 (SD 9.42) completed interventions per driver, with a minimum and maximum of 17 and 49, respectively. In total, 138 mindfulness and 175 music interventions have been completed. The average trip lasted 36.1 minutes (SD 21.8 minutes). Participants on average initiated the intervention 2.6 minutes (SD 7.1 minutes) after the start of their trip. The remaining driving time after the intervention was on average 20.5 minutes (SD 19.9 minutes). These numbers illustrate two important facts: a) on average, the participants had enough time to complete the interventions and b) our data include uninstructed real-world trips (trip duration varies considerably). Figure 4(a) shows the difference in trip duration throughout the

\footnotetext{
${ }^{1}$ The natural logarithm is applied because we assume non-linear effects.
}

study, while Figure 4(b) indicates that the trips of a typical driver covered more than the daily commute.

5.1.2 Mindfulness. As a first step in our data analysis, we visualize the immediate impact of the mindfulness intervention on affect ( 0 = "no improvement at all" to 4 = "very strong improvement") for an initial visual impression. Figure 5 depicts trends of the immediate intervention impact across all participants in increasing time granularity (a: over the entire study, b: by weekday, and c: over the course of the day). While the smoothed mean ratings of the participants (from 0 ("no improvement at all") to 4 ("very strong improvement")) range from 0.5 to 2 , in most cases, they remain close to 1 . In general, the mindfulness intervention had a slightly higher immediate impact on arousal than on valence. In Figure 5(a), the immediate intervention impact for arousal and valance first decreases over time until it reaches a maximum after approximately 20 days into the study before it declines again and ends roughly at the initial level, hence indicating a slight negative trend after 25 days. Figure 5(b) shows that the immediate impact of the intervention on arousal and on valence is rather stable over the course of the week, with a minor arousal low in the middle of the week. Figure 5(c) illustrates an increase in the impact of the mindfulness intervention on both arousal and valence over the course of the day, with an intermediate spike in the morning hours, when people most likely commuted to work.

Next, we investigate whether these visual trends of the immediate impact on affect can be validated on the basis of our hypotheses H1 - H5. The results of the mixed-effect models are summarized in Table 4. All models have an intercept significantly different from 
Table 4: Statistical results. Immediate intervention impact on arousal and valence; significance levels are indicated as ${ }^{* *}: p<0.01$, ${ }^{*}: \mathrm{p}<0.05$, and ${ }^{\wedge}: \mathrm{p}<0.1$

Immediate impact on affect of interventions

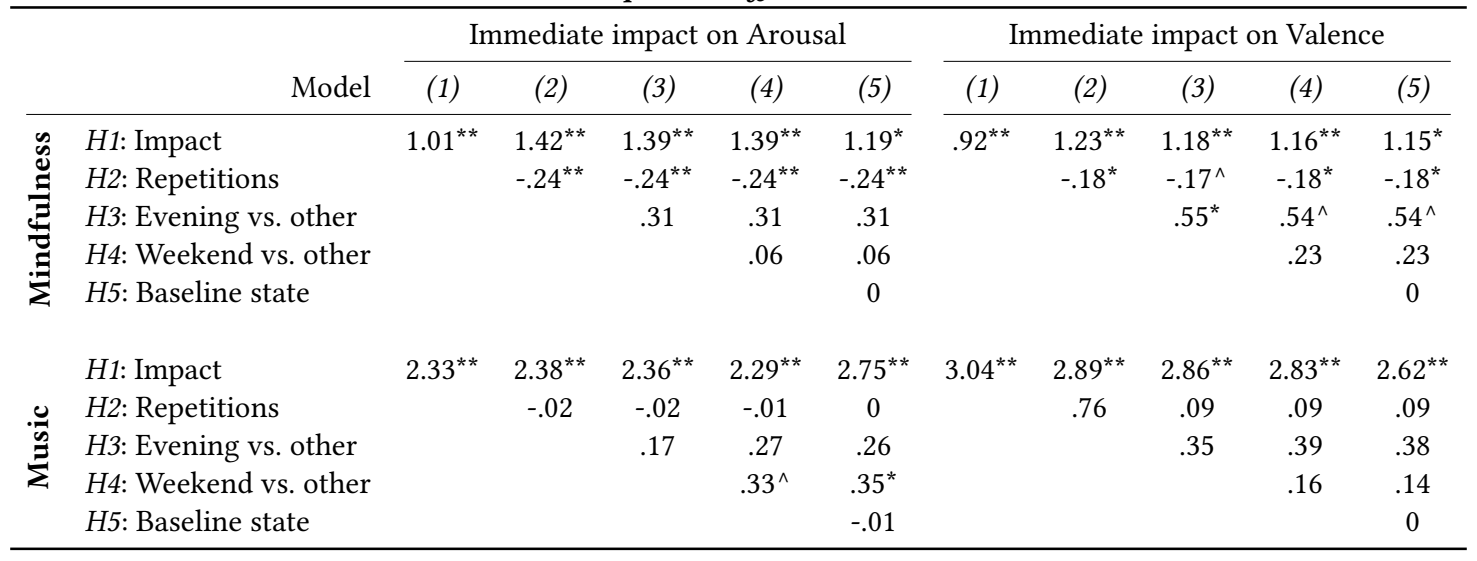

0 (i.e., immediate intervention impact "no improvement at all"). The first two models reveal a significant immediate impact of the mindfulness intervention both on arousal $(p=0.004, b=1.01)$ and valence ( $p=0.025, b=0.92)$. Hence, the results imply that the mindfulness intervention has an immediate impact on the affect of drivers and thus support $H 1$. The mixed-effect models also confirm the negative trend over time visible in Figure 5(a). The first models of arousal and valence that include the repetition variable reveal a negative significant coefficient for both arousal $(p=0.003$, $b=-0.24)$ and valence ( $p=0.041, b=-0.18)$. Consequently, we reject $H 2 a$; in fact, the effect goes in the opposite direction, indicating a diminishing immediate impact of the mindfulness intervention on affect with more repetitions. Figure 5(c) reveals that in the evening hours, the mindfulness intervention is significantly more effective than over the rest of the day. However, this effect is limited to valence $(p=0.050, b=0.55)$. In contrast, the effect on arousal is non-significant. We therefore find limited support for H3. Similar to the descriptive results visualized in Figure 5(b), the mixed-effects models show no significant difference between weekdays and weekend ( $H 4)$. In addition, we find no evidence that the pre-driving affective state has a significant influence on the drivers' perceived immediate impact on affect and thus reject $H 5$.

To understand the persistence of the impact of the mindfulness intervention, we analyze how the post-driving state depends on the pre-driving (baseline) state and the improvement induced by the intervention (immediate impact). The analysis shows a significant positive influence of the immediate impact on post-driving valence $(p=0.015, b=2.86)$. Thus, the higher the participants perceived their valence improvement directly after the intervention, the higher their post-driving valence state. While the point estimate for the immediate impact on arousal is positive $(b=1.66)$, the result is not significant. Based on these results, we see partial support for H6. The results also stress the importance of including the baseline state in the model. The baseline state before driving has a significant influence on the respective state after driving (in both cases, $p<0.001$ and point estimates ranging from 0.43 to
Table 5: Statistical results. Effects of interventions on arousal and valence post-driving affective state; significance levels are indicated as ${ }^{* *}: \mathrm{p}<0.01,{ }^{*}: \mathrm{p}<0.05$, and ${ }^{\wedge}: \mathrm{p}<0.1$

Impact on post-driving state of interventions

\begin{tabular}{|c|c|c|c|c|}
\hline & \multicolumn{2}{|c|}{ Mindfulness } & \multicolumn{2}{|c|}{ Music } \\
\hline & Arousal & Valence & Arousal & Valence \\
\hline Constant & $28.92^{* *}$ & $36.80^{* *}$ & $33.93^{* *}$ & $31.78^{* *}$ \\
\hline Baseline state & $.54^{* *}$ & $.43^{* *}$ & $.50^{* *}$ & $.49^{* *}$ \\
\hline H6: Immediate impact & 1.66 & $2.86^{*}$ & .55 & $1.78^{\wedge}$ \\
\hline
\end{tabular}

0.54). In other words, the higher the driver's pre-driving arousal (valence), the higher the driver's post-driving arousal (valence).

For the music intervention, we perform the same steps of analysis as for the mindfulness intervention. Figure 6 provides a visual impression of the immediate impact of the music intervention on affect, as perceived by the participants. Compared to the mindful intervention, the immediate impact ratings are considerably higher, with values ranging between 2 and 3.5 (compared to 0.5 to 2). Over the entire study (Figure 6(a)), the intervention exhibits a positive time trend both for arousal and valence. With respect to the development over the week, Figure 6(b) suggests a small increase towards the weekend. Regarding daytime, Figure 6(c) indicates an increase towards the end of the day.

To investigate the immediate impact of the music intervention thoroughly, we evaluate multiple model specifications. As Table 4 shows, the results indicate a significant immediate impact both on arousal and valence in all model specifications. The first arousal model (i.e., main effect alone) has an intercept of 2.33, which reflects a medium impact on affect (impact was measured on a scale between 0 and 4). The first valence model even has the intercept at 3.04, reflecting a strong immediate improvement of valence. Given these results, we fail to reject $H 1$ and thus conclude that the music intervention improves immediate arousal and valence of drivers. 
(a)
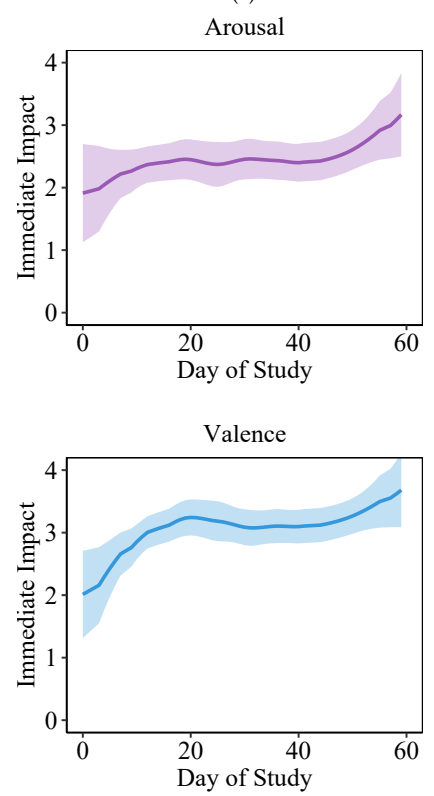

(b)
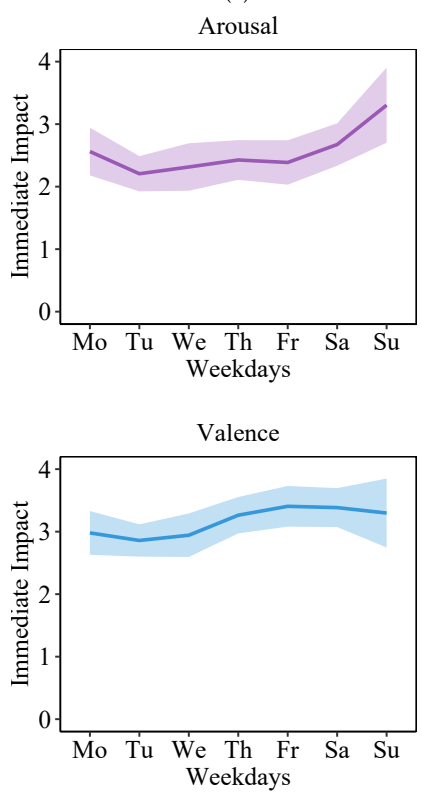

(c)
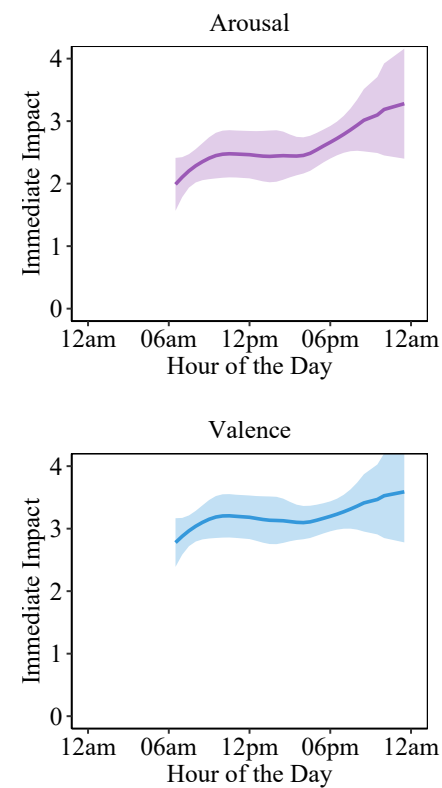

Figure 6: Immediate impact of the music intervention on arousal (upper row) and valence (lower row). The graphs show the loess-smoothed mean value and the 5 to 95 percentiles for $n=175$ completed music interventions. (a) effects over the study time; (b) effects over the weekdays; and, (c) effects over the day.

Although the visual trends in Figure 6(a) is suggestive of increasing intervention effects over time, we do not find significant effects in our regression analysis to support $\mathrm{H} 2 b$. Likewise, contrary to the visual impression in Figure 6(c), the mixed models do not reveal significantly stronger effects in evening hours (H3). However, we find stronger immediate effects of the music intervention on weekends for arousal $(p=0.052, b=0.33)$. Although the valence model has no significant effect, the coefficient $(b=0.16)$ indicates a potential positive effect. The plots in Figure $6(\mathrm{~b})$ are in line with these findings. Hence, we find evidence for $\mathrm{H} 4$ regarding arousal. Similar to the mindfulness intervention, the immediate impact of the music intervention does not correlate with pre-driving (baseline) affect. Hence, we find no support for $\mathrm{H} 5$ and thus no evidence for the assumption that a lower pre-driving state results in a larger immediate impact of the intervention.

With respect to the effects of the music intervention on the post-driving state, the results in Table 5 reveal similar patterns as for the mindfulness intervention. Concerning valence, the music intervention's immediate impact has a positive and significant effect on the post-driving state ( $p=0.077, b=1.78$ ). For arousal, the corresponding coefficient is insignificant, but points in the positive direction $(b=0.55)$. Hence, we find partial support for the impact of the the music intervention on the post-driving state (H6). In addition, the results confirm the significant positive effect of the baseline (pre-driving) state on the post-driving state, in line with the results for the mindfulness intervention.

\subsection{Qualitative Analysis and Results}

At the end of the study, we conducted semi-structured interviews of approximately one hour with each of the ten participants to better understand the interventions and their effects. We recorded the interviews and transcribed them for our analysis. This section outlines the main insights for the two interventions.

5.2.1 Mindfulness Intervention. The interviews reveal an ambivalent perception of the mindfulness intervention, especially in the car. Either people experience it as helpful or not.

Perceived Effects. In total, four participants were positive towards the exercise (P-2, P-5, P-9, and P-10), independent of prior experience. These participants perceived the intended benefits of the exercise (e.g., "a helicopter perspective on the situation", "reflect more about myself", "how do I react to situations", "better manage the rude behavior of other traffic participants"). As a consequence, they felt the support given by our intervention to be "centered and calm" and "to feel better". These statements support H1. In contrast, the remaining six participants struggled with the mindfulness exercise in the car. Both P-6 and P-7, with previous mindfulness experience, share the opinion that other places such as "a chair, a sofa or even in bed" or "after a yoga workout" are more appropriate. The other participants were simply "not convinced of the concept" and therefore did not perceived it as helpful. Although four participants had a positive mindset towards the exercise, they agreed that "it was very repetitive and therefore the effect has decreased over time", which is in contrast to H2a. In fact, after several runs, all participants shared a similar experience about the missing variance of the mindfulness intervention (e.g., "always the same text", "got a 
little bit on my nerves because it was the same thing over and over"). Lastly, all participants mentioned the length of the mindfulness exercise, especially in the case of shorter trips. They would have favored a shorter version.

Distraction and Safety. During the interviews, the drivers rated the intervention's distraction from driving on a scale from $1=$ "not at all" to 5 = "very much", which yielded an average rating of 2.1 $(\mathrm{SD}=1.5)$. The average is rather low, however the standard deviation indicates different perceptions. The participants reported that their concentration and thus the effectiveness of the interventions depends on the traffic. While they drove on straight streets or with less traffic (e.g., motorway), they "could concentrate well on the exercise". They "had to concentrate more on the traffic" in situations that require frequent driver interactions, e.g., in city traffic. In this context, two potential safety risks appeared. Four participants mentioned that it was sometimes too demanding to listen to the exercise in addition to the driving task. In all these cases they just concentrated on the street and simply ignored the intervention. However, two participants felt "extremely distracted at times". Three participants claimed that the exercise "was rather lulling to sleep". Two of them named drowsiness a common problem they experience while driving. In contrast, P-2, who perceived himself as an "aggressive driver", reported a beneficial effect. Because of our intervention, he felt that he was driving more calmly.

Future Use. The four participants sharing a positive attitude about the exercise declared that they would continue to carry out the intervention, but they mentioned a strong need for more variation. One participant "would rather do it on demand and when the car is stationary". The remaining participants are unlikely to do so because they do not believe in mindfulness exercises.

5.2.2 Music Intervention. Seven out of ten participants preferred the music intervention to the mindfulness intervention. Nine participants were convinced that the music intervention often improved their well-being. The only exception is P-8, who struggled with the music intervention. By the time he had created his personal playlist, he admitted that he "hardly ever associated his positive memories with music" and that he is generally "not a music type".

Perceived Effects. In addition to the effects of the music alone, eight participants mentioned that recalling their emotions positively influenced their perception (e.g., "The positive feelings associated with the music were enjoyable.", "I was happier", "Memories came up again and again and that always put you in a good mood.") because the intervention "always played [their] favorite music". These results support that the music intervention improved the affect (H1). The participants mostly reported on stable effects of the music interventions over time that are opposed to our initial expectations $(H 2 b)$. A majority perceived "the same [effects] most of the time". However, three participants experienced decreasing effects or at least minor effects towards the end. They would have "liked to add and to remove certain songs after some time" to keep the playlist enjoyable.

Distraction and Safety. Participants rated the intervention's distraction from driving ( $1=$ "not at all" to $5=$ "very much") as very low. They evaluated the question with an average rating of 1.3
$(\mathrm{SD}=0.5)$. The participants could easily drive and experience the intervention at the same time. They attribute it to the possibility to quickly change their attention between the music and back to the street (e.g., "it's just that sometimes you don't have to listen").

Future Use. Nine of ten participants would keep using the intervention in their car. The participants perceived the music intervention as positive "because it is was [their] playlist" and they felt "self-controlled and not controlled by others". In addition, they preferred the easy accessibility of their favourite songs to the sometimes cumbersome playback over their telephone or the absence of the songs on the radio (e.g., "just a single button and not 5 clicks", "[the songs] are often not on the radio"). Their enthusiasm went so far that four participants already made suggestions on how such a system could be implemented in the car (e.g., "The car should learn my favorite songs based on what I listen to.", "I recommend a direct connection between Spotify and the intervention.").

\section{DISCUSSION AND FUTURE WORK}

This article presents the first real-world driving study on in-vehicle interventions for mental health and well-being for daily commuters. In this section, we discuss our findings and derive design recommendations that both researchers and industry can use as a starting point for developing future in-vehicle interventions.

\subsection{Impact of In-Vehicle Interventions}

The primary aim of our study was to understand if, when and, how in-vehicle well-being interventions have an impact on daily drivers. We have developed two interventions adapted to the invehicle context and used an in-situ feedback tool to investigate their fundamental mechanisms and drivers' interactions with them in the real world. Our study provides empirical evidence that invehicle interventions have the potential to improve the well-being of everyday commuters. Our quantitative analysis reveals positive effects both for the music and mindfulness intervention. However, as the results of the qualitative analysis in particular reveal, the two interventions differ in their impact and assessment by the participants, hence a more nuanced interpretation is necessary. Thereby, we can derive general guidelines for the effective design of in-vehicle well-being interventions. In addition, we are able to share our experience in conducting longitudinal studies of open road driving.

6.1.1 General Impact of In-Vehicle Interventions. Based on the results of our quantitative analysis, both interventions improve the affect of drivers (H1) and therefore their well-being. Notably, the interventions even indicate positive effects on the post-driving state of drivers (H6). While the effects of the mindfulness intervention declined over the course of the study, the effects of the music intervention were stable. Based on these results, we provide real-world evidence that in-vehicle interventions embedded in a necessary daily task can indeed increase individuals' well-being. However, we also find that well-proven psychotherapeutic measures such as the mindfulness exercise need to be adapted to the specific requirements of in-vehicle environments. Bringing mental health and well-being interventions into the car is thus much more than 
simply "moving" established best practice approaches into a new environment.

6.1.2 Mindfulness Intervention. Our findings suggest a significant impact of the mindfulness exercise on affect. However, we believe that the potential of in-vehicle mindfulness interventions is larger than our quantitative results suggest. The qualitative results revealed that four of the ten participants adopted the mindfulness intervention and considered it as helpful. In contrast, the other participants named two common barriers for a successful adoption. Drivers must be able a) to handle the cognitive load of a mindfulness intervention while driving and $b$ ) to relate to the concept of mindfulness. In order to keep the cognitive load of the mindfulness intervention low, several design opportunities should be considered. For example, the overall intervention can be shortened and the pauses within the intervention can be extended. In essence, a very diligent and careful design seems to be necessary to deliver an effective mindfulness intervention with an appropriate cognitive load in the in-vehicle environment. Further, people must be open to the concept of mindfulness to experience benefits. Additional explanations illustrating the purpose and effect of such exercises might potentially help to increase acceptance. Another important concern that all participants named was the lack of variety. Already after a few number of interventions, participants perceived the exercise as monotonous and boring. While existing literature indicates that several repetitions of the same mindfulness exercise will improve its effects (e.g., [36]), our findings stress the need for change. Summing up, we strongly believe mindfulness exercises can be an effective instrument in the car. We have just inspected one particular mindfulness exercise that already had a significant positive immediate impact and the results even indicate a positive post-driving impact. While mindfulness exercises in the in-vehicle environment may not be a suitable instrument for everyone, we still conjecture that there is a target group that can benefit from such interventions.

6.1.3 Music Intervention. Our results clearly demonstrate the power of emotional music interventions that are delivered in the vehicle for mental health and well-being. Nine out of the ten drivers stated they had a positive experience with the intervention. Only one study participant reported that he had difficulties in linking positive emotional memories with music, and thus expressed a more negative opinion. The participants attributed their positive experiences with the music intervention to two factors: emotions and accessibility. The connection to emotions helped them to choose very personal songs tied to positive memories and the intervention offered them a very convenient way to access this personalized music selection. Notably, the music intervention had a stable impact on affect over the total study duration of two months. Hence, we were not able to confirm an inverse U-shaped intervention effect over time as described in prior research [14]. However, our intervention period was limited to two months and deteriorating effects could materialise later. Besides, the intervention varied substantially with at least ten different songs for each participant. The participants who reported decreasing effects attributed this mostly to their limited choice of songs. To keep the music intervention effective for even longer periods, participants should be able to update their playlists. At the same time, our results suggest that there is no strong need for very frequent updating.

6.1.4 Timing of In-Vehicle Interventions. Existing empirical evidence has led us to hypothesize that there are different points in time when the impact of interventions is particularly large [49]. Identifying and leveraging such favorable moments is an important part of intervention delivery [41]. Our statistical analysis suggests evenings (H3) and weekends (H4) to be particularly promising. In the evening, we observed higher effects, particularly for the mindfulness exercise. The music intervention achieved a higher impact on weekend days than weekdays. We conjecture that the type of the intervention plays a role in the time-related effects. For instance, most people have more free time on weekends and therefore they have less on their mind in comparison to a weekday [32]. Hence, they might be more relaxed and better able to recall positive emotions on the basis of the music intervention. However, we see the necessity for future research to shed light on the interaction between intervention type and delivery time. Although we provide first actionable insights when to deliver interventions (i.e., in the evening and on weekends), future research should also reflect on "state of receptivity" and "state of vulnerability" [49], i.e., to understand whether in-vehicle intervention impact is achieved because the driver is more receptive (e.g., time available for intervention) or because there is a larger need for treatment.

6.1.5 Distraction and Safety. In-vehicle interventions can only be applied if they do not compromise driving safety. On average, our participants rated the distraction caused by the interventions as low or very low. While there were absolutely no safety concerns with the music intervention, two potential issues were named for the mindfulness exercise. First, six of the ten participants indicated cognitive overload, i.e., combining mindfulness practice with driving exceeds existing mental capabilities. We believe that for the active participation in the exercise a better understanding of the driving context is required. More specifically, less demanding situations, e.g., clear road conditions with low traffic density, are promising situations for the intervention, which is is in line with existing literature (e.g., [60]). Second, three participants indicated that the mindfulness practice might intensify drowsiness while driving. Therefore, intervention delivery should consider situational and driver-specific factors.

6.1.6 Design Recommendations. Based on the findings of this study, other researchers or practitioners might consider the following design recommendations for future in-vehicle interventions. First, we recommend investigating variations of interventions. On the basis of our participants' experiences, we are able to derive explicit design considerations. For example, if we consider mindfulness, we can imagine changing the following parameters of the exercise: Content, length, use of pauses, rhythm of speech or the gender of the narrator. Our results indicate that a certain level of novelty and variation are vital for longevity, frequent use, and acceptance. Second, we believe that it is necessary to design a set of different intervention types from which drivers can choose. We implemented two distinct interventions that significantly increase drivers' wellbeing and learned that drivers perceived them quite differently. While most drivers enjoyed the music intervention, participants 
found the mindfulness exercise only helpful in specific situations. In-vehicle interventions might reach a broader spectrum of users when addressing specific needs. While some drivers may need assistance in overcoming drowsiness, others may want to relax more in order to drive less aggressively. In this context, an important lesson from our study is that the simple transfer of established well-being concepts to the car is not enough. Instead, careful adaptation of proven means is necessary. Finally, context-aware systems tailored to the driver and road conditions would ensure that the most effective intervention is delivered at the right time, under safe conditions, and adapted if necessary. Examples include an arousing intervention in case of driver drowsiness or the suppression or cancellation of demanding intervention in stressful driving situations. We provide first evidence for the power of context-aware systems and identify evenings and weekends as rather promising times for interventions.

6.1.7 Longitudinal On-Road Studies. Open road driving studies are challenging, and previous work has not tested in-vehicle wellbeing interventions in a field study over several months for good reasons (e.g., due to the high safety requirements and substantial costs involved). However, we strongly believe that studying the basic effects and mechanisms in a real environment (rather than in a simulator or a controlled environment) is critical to achieve progress towards real-world applications. Therefore, we share our experiences and best practices to pave the way for $\mathrm{HCI}$ researchers to conduct similar longitudinal studies on open roads:

- Safety first. In our case, there was little knowledge about invehicle interventions in unrestricted situations. As a consequence, we gave drivers full control over the interventions (start, stop, pause) as they can decide best when they are appropriate or not. Moreover, in light of driver safety, we focused on fundamental interactions. In the future, more sophisticated and powerful interaction strategies should be explored. However, they always have to be assessed in the light of driver safety first.

- Keep the system simple and robust. We identified the core interactions that we wanted to investigate and assured that they were rigorously tested and error-free. During the study, we could only have fixed errors remotely as the drivers lived across a large area. Even remote fixes can have a substantial effect on the overall study and should be avoided if possible.

- Leverage existing vehicle interfaces. Vehicle manufacturers prevent access to their in-vehicle software systems, which discourages many researchers. Our approach shows that no modification of any car software is needed. We simply used a smartphone with an app connected to the infotainment system via a well-defined interface (Bluetooth) to introduce our in-vehicle interventions.

- Test the interventions under real-world conditions. We learned that testing our setup at the desk or in a stationary vehicle alone is not enough. The perception of interventions is fundamentally different in real traffic situations. Hence, we had two drivers test our study setup for several days before we actually started the study. The problems discovered and solved in this pilot phase gave us confidence that the study could run over a longer period of time.

- Use in-situ voice feedback while driving. Asking drivers and recording their answers is a convenient and safe way to understand their current perceptions and intentions. We have collected feedback on drivers' emotional states, but a voice assistant-based questionnaire could also cover a much broader range of aspects including driver distraction or workload.

- Monitor the data collection. Because we had no physical access to the cars throughout the study, we had routines in place to monitor the data collection. More specifically, we implemented a $24 / 7$ dashboard to visualize our data collection in real-time. This helped us tremendously to identify errors early on.

\subsection{Limitations}

Despite our best efforts, this study has several limitations. First, the designed interventions largely differ in their concepts and are conducted in real-world traffic, a very complex environment. Hence, results may be subject to a combination of several observed and unobserved factors, which future studies need to disentangle. Second, given the cost associated with the prototypical setup, we had to limit our sample size to ten drivers. At the same time, the study is the first of its kind on the road covering a longer period of time. Therefore, it complements prior research in controlled settings and thus represents a starting point for empirical research on in-vehicle interventions under realistic conditions. Third, we used questionnaires and interviews to evaluate the perceived impact of our interventions in the vehicle. While we believe that our results are already very comprehensive, future studies may consider further measurements such as heart rate to provide additional insights on subtle changes that may complement the drivers' subjective ratings. Fourth, our study was conducted prior to the Corona outbreak. Although the post-pandemic world will be different, we believe that the car will retain its relevance. Presence during the pandemic was required in many occupations and will be required again in many others. With the upcoming vaccination routines, commuting will normalize, albeit perhaps at a lower level. Finally, while the opinions and statements of the participants are promising, they are no safety experts. Before the mass roll-out of an intervention is considered for application in a future car generation, more rigorous safety inspections (i.e., lane keeping tests or gaze tracking) are highly recommended.

\section{CONCLUSION}

The aim of this study was to support ordinary everyday commuters by improving their mental health and well-being through the use of established psychotherapy concepts during their commutes. In particular, it was not at all clear whether existing well-being interventions could be successfully transferred to the car. More specifically, prior work has not tested in-vehicle well-being interventions in a real-life field study over multiple months for good reasons. It is high-risk research (general challenge to find significant effects in the field) associated with substantial costs (costs of renting a fleet of identical cars to provide a homogeneous intervention setting). To that end, we conducted the first longitudinal in-vehicle intervention study on public roads. During a period of two months, ten drivers were randomly exposed to two different in-vehicle interventions, a mindfulness exercise and an emotional music experience. More specifically, we sought to determine to which extent interventions in the vehicle have a) an immediate impact on drivers' affect and 
b) an impact on drivers' post-driving affect. We explored affect as the dependent variable, as past research has identified affect as a cornerstone of well-being and mental health.

Our results show that (1) both tailored in-vehicle interventions (2) significantly increase drivers' well-being (3) over an extended time period (4) without compromising drivers' safety. Furthermore, our analyses of the timing (daytime and weekday of delivery) and context (repetition and pre-intervention driver state) provide a concrete and actionable foundation (5) when to interact with drivers allowing for more advanced strategies with a higher degree of user interaction. Moreover, we derive general guidelines (6) for the effective design of such interventions and implications for future $\mathrm{HCI}$ research. Prior work suggests that the mindfulness intervention ("gold standard" in psychotherapy) should outperform the music intervention, particularly over time. Our work reveals the opposite. Hence, we deduct actionable implications for the design of in-vehicle interventions. Finally, the methodology of our study can serve as a blueprint for future driving studies to capture driver interactions in unconstrained environments. We provide specific recommendations on how to conduct in-vehicle measurements and open road experiments over a longer period of time.

In summary, we conclude that in-vehicle interventions are indeed promising tools for improving mental health and well-being among ordinary daily commuters. On the basis of two exemplary types of interventions, our results illustrate the challenges of bringing helpful interventions into the car, while at the same time providing specific insights that research and industry can use for further developing in-vehicle interventions.

\section{ACKNOWLEDGMENTS}

This research was funded and supported by the Bosch IoT Lab of the University of St. Gallen and ETH Zürich. Additional support for the evaluation of the study was provided by the Bavarian State Ministry of Science and the Arts (coordinated by bidt).

\section{REFERENCES}

[1] American Psychiatric Association. 2013. Diagnostic and Statistical Manual of Mental Disorders (DSM-5). American Psychiatric Association, Washington, DC.

[2] Audi AG. 2016. Audi Fit Driver. https://www.audi-mediacenter.com/en/audi-atthe-ces-2016-5294/audi-fit-driver-5300 Accessed: 09/03/2020.

[3] Ruth A. Baer. 2003. Mindfulness Training as a Clinical Intervention: A Conceptual and Empirical Review. Clinical Psychology: Science and Practice 10, 2 (2003), 125143.

[4] Stephanie Balters, Matthew L. Mauriello, So Yeon Park, James A. Landay, and Pablo E. Paredes. 2020. Calm Commute: Guided Slow Breathing for Daily Stress Management in Drivers. Proceedings of the ACM on Interactive, Mobile, Wearable and Ubiquitous Technologies 4, 1, Article 28 (2020), 19 pages.

[5] Judith S. Beck and Aaron T. Beck. 1995. Cognitive Therapy: Basics and Beyond Guilford press, New York, NY, USA

[6] Klaus Bernhardt. 2019. Depression und Burnout loswerden: Wie seelische Tiefs wirklich entstehen, und was Sie dagegen tun können. Ariston, Munich, Germany.

[7] Alberto Betella and Paul F. M. J. Verschure. 2016. The Affective Slider: A Digital Self-Assessment Scale for the Measurement of Human Emotions. PLoS ONE 11, 2, Article e0148037 (2016), 11 pages

[8] Lynn Boschloo, Pim Cuijpers, Eirini Karyotaki, Thomas Berger, Steffen Moritz, Björn Meyer, and Jan Philipp Klein. 2019. Symptom-specific effectiveness of an internet-based intervention in the treatment of mild to moderate depressive symptomatology: The potential of network estimation techniques. Behaviour Research and Therapy 122, Article 103440 (2019), 7 pages.

[9] Sophie Bostock, Alexandra D. Crosswell, Aric A. Prather, and Andrew Steptoe. 2019. Mindfulness on-the-go: Effects of a mindfulness meditation app on work stress and well-being. Fournal of Occupational Health Psychology 24, 1 (2019), 127-138.
[10] Margaret M. Bradley and Peter J. Lang. 1994. Measuring emotion: The selfassessment manikin and the semantic differential. Fournal of Behavior Therapy and Experimental Psychiatry 25, 1 (1994), 49-59.

[11] Michael Braun, Anja Mainz, Ronee Chadowitz, Bastian Pfleging, and Florian Alt. 2019. At Your Service: Designing Voice Assistant Personalities to Improve Automotive User Interfaces. In Proceedings of the Conference on Human Factors in Computing Systems (CHI). Article 40, 11 pages.

[12] Philip Brickman and Donald T. Campbell. 1971. Hedonic relativism and planning the good society. Academic Press, New York, NY, USA.

[13] Rafael A. Calvo and Sidney D'Mello. 2010. Affect Detection: An Interdisciplinary Review of Models, Methods, and Their Applications. IEEE Transactions on Affective Computing 1, 1 (2010), 18-37.

[14] Anthony Chmiel and Emery Schubert. 2017. Back to the inverted-U for music preference: A review of the literature. Psychology of Music 45, 6 (2017), 886-909.

[15] Daimler AG. 2018. ENERGIZING comfort control: Wellness while driving. https: //media.daimler.com/marsMediaSite/ko/en/41880672 Accessed: 09/03/2020.

[16] Daniel David, Ioana Cristea, and Stefan Hofmann. 2018. Why Cognitive Behavioral Therapy Is the Current Gold Standard of Psychotherapy. Frontiers in Psychiatry 9, Article 4 (2018), 3 pages. https://doi.org/10.3389/fpsyt.2018.00004

[17] Ed Diener, Shigehiro Oishi, and Louis Tay. 2018. Advances in subjective wellbeing research. Nature Human Behaviour 2, 4 (2018), 253-260.

[18] Ed Diener, Sarah D. Pressman, John Hunter, and Desiree Delgadillo-Chase. 2017. If, Why, and When Subjective Well-Being Influences Health, and Future Needed Research. Applied Psychology: Health and Well-Being 9, 2 (2017), 133-167.

[19] Ed Diener, Eunkook M. Suh, Richard E. Lucas, and Heidi L. Smith. 1999. Subjective well-being: Three decades of progress. Psychological Bulletin 125, 2 (1999), 276302

[20] Harvey Dillon, Alison James, and Jenny Ginis. 1997. Client Oriented Scale of Improvement (COSI) and its Relationship to Several Other Measures of Benefit and Satisfaction Provided by Hearing Aids. Fournal-American Academy of Audiology 8 (1997), 27-43.

[21] Dmitrijs Dmitrenko, Emanuela Maggioni, Giada Brianza, Brittany E. Holthausen, Bruce N. Walker, and Marianna Obrist. 2020. CARoma Therapy: Pleasant Scents Promote Safer Driving, Better Mood, and Improved Well-Being in Angry Drivers. In Proceedings of the Conference on Human Factors in Computing Systems (CHI). Article 49, 13 pages.

[22] David Daniel Ebert, Pim Cuijpers, Ricardo F. Muñoz, and Harald Baumeister. 2017. Prevention of Mental Health Disorders Using Internet- and Mobile-Based Interventions: A Narrative Review and Recommendations for Future Research. Frontiers in Psychiatry 8, Article 116 (2017), 16 pages. https://doi.org/10.3389/ fpsyt.2017.00116

[23] Tammy English and Laura Carstensen. 2014. Emotional experience in the mornings and the evenings: consideration of age differences in specific emotions by time of day. Frontiers in Psychology 5, Article 185 (2014), 9 pages. https://doi.org/10.3389/fpsyg.2014.00185

[24] Giovanni A. Fava and Elena Tomba. 2009. Increasing Psychological Well-Being and Resilience by Psychotherapeutic Methods. Fournal of Personality 77, 6 (2009), 1903-1934.

[25] Kathleen Kara Fitzpatrick, Alison Darcy, and Molly Vierhile. 2017. Delivering cognitive behavior therapy to young adults with symptoms of depression and anxiety using a fully automated conversational agent (Woebot): a randomized controlled trial. FMIR mental health 4, 2, Article e19 (2017), 11 pages.

[26] Charlotte Fritz, Sabine Sonnentag, Paul E Spector, and Jennifer A McInroe. 2010. The weekend matters: Relationships between stress recovery and affective experiences. Fournal of Organizational Behavior 31, 8 (2010), 1137-1162.

[27] Paul E. Greenberg, Andree-Anne Fournier, Tammy Sisitsky, Crystal T. Pike, and Ronald C. Kessler. 2015. The economic burden of adults with major depressive disorder in the United States (2005 and 2010). Fournal of Clinical Psychiatry 76, 2 (2015), 155-162.

[28] Jenny Gu, Clara Strauss, Rod Bond, and Kate Cavanagh. 2015. How do mindfulness-based cognitive therapy and mindfulness-based stress reduction improve mental health and wellbeing? A systematic review and meta-analysis of mediation studies. Clinical Psychology Review 37 (2015), 1-12.

[29] Helen Harris and Clifford Nass. 2011. Emotion regulation for frustrating driving contexts. In Proceedings of the Conference on Human Factors in Computing Systems (CHI). 749-752.

[30] Steven C. Hayes and Stefan G. Hofmann. 2018. Process-based CBT: the science and core clinical competencies of cognitive behavioral therapy. New Harbinger Publications, Oakland, CA, USA.

[31] Andrew Hede. 2010. The dynamics of mindfulness in managing emotions and stress. Journal of Management Development 29, 1 (2010), 94-110.

[32] John F. Helliwell and Shun Wang. 2014. Weekends and Subjective Well-Being. Social Indicators Research 116, 2 (2014), 389-407.

[33] Javier Hernandez, Daniel McDuff, Xavier Benavides, Judith Amores, Pattie Maes, and Rosalind Picard. 2014. AutoEmotive: Bringing Empathy to the Driving Experience to Manage Stress. In Proceedings of the 2014 Companion Publication on Designing Interactive Systems. 2602780, 53-56. 
[34] Annika Howells, Itai Ivtzan, and Francisco Jose Eiroa-Orosa. 2016. Putting the 'app'in happiness: a randomised controlled trial of a smartphone-based mindfulness intervention to enhance wellbeing. Fournal of Happiness Studies 17, 1 (2016), 163-185.

[35] Lutz Jäncke. 2008. Music, memory and emotion. fournal of Biology 7, 6, Article 21 (2008), 5 pages

[36] Jon Kabat-Zinn. 1982. An Outpatient Program in Behavioral Medicine for Chronic Pain Patients Based on the Practice of Mindfulness Meditation: Theoretical Considerations and Preliminary Results. General Hospital Psychiatry 4, 1 (1982), 33-47.

[37] Jon Kabat-Zinn. 1994. Wherever You Go, There You Are: Mindfulness Meditation for Everyday Life. Hyperion, New York, NY, USA.

[38] Robert Kohn, Shekhar Saxena, Itzhak Levav, and Benedetto Saraceno. 2004. The treatment gap in mental health care. Bulletin of the World Health Organization 82, 11 (2004), 858-866.

[39] Tobias Kowatsch, Fabian Wahle, and Andreas Filler. 2017. stressOUT: Design Implementation and Evaluation of a Mouse-based Stress Management Service. In Designing the Digital Transformation: DESRIST 2017 Research in Progress Proceedings. $37-45$.

[40] Alexandra Kuznetsova, Per B. Brockhoff, and Rune H. B. Christensen. 2017 lmerTest Package: Tests in Linear Mixed Effects Models. Journal of Statistical Software 82, 13 (2017), 1-26.

[41] Florian Künzler, Varun Mishra, Jan-Niklas Kramer, David Kotz, Elgar Fleisch, and Tobias Kowatsch. 2019. Exploring the State-of-Receptivity for mHealth Interventions. Proceedings of the ACM on Interactive, Mobile, Wearable and Ubiquitous Technologies 3, 4, Article 140 (2019), 27 pages.

[42] Daniel Leubner and Thilo Hinterberger. 2017. Reviewing the Effectiveness of Music Interventions in Treating Depression. Frontiers in Psychology 8, Article 1109 (2017), 21 pages. https://doi.org/10.3389/fpsyg.2017.01109

[43] Emily Lynar, Erin Cvejic, Emery Schubert, and Ute Vollmer-Conna. 2017. The joy of heartfelt music: An examination of emotional and physiological responses. International Journal of Psychophysiology 120 (2017), 118-125.

[44] Elizabeth Hellmuth Margulis. 2014. On repeat: How music plays the mind. Oxford University Press, New York, NY, USA.

[45] Nikolas Martelaro, Sarah Mennicken, Jennifer Thom, Henriette Cramer, and Wendy Ju. 2020. Using Remote Controlled Speech Agents to Explore Music Experience in Context. In Proceedings of the ACM Designing Interactive Systems Conference. 2065-2076.

[46] Nikolas Martelaro, Jaime Teevan, and Shamsi T. Iqbal. 2019. An Exploration of Speech-Based Productivity Support in the Car. In Proceedings of the Conference on Human Factors in Computing Systems (CHI). Article 264, 12 pages.

[47] Aire Mill, Anu Realo, and Jüri Allik. 2016. Retrospective Ratings of Emotions the Effects of Age, Daily Tiredness, and Personality. Frontiers in Psychology 6, Article 2020 (2016), 12 pages. https://doi.org/10.3389/fpsyt.2018.00004

[48] Daniel Mochon, Michael I. Norton, and Dan Ariely. 2008. Getting off the hedonic treadmill, one step at a time: The impact of regular religious practice and exercise on well-being. Fournal of Economic Psychology 29, 5 (2008), 632-642.

[49] Inbal Nahum-Shani, Eric B. Hekler, and Donna Spruijt-Metz. 2015. Building health behavior models to guide the development of just-in-time adaptive interventions: A pragmatic framework. Health psychology 34 (2015), 1209-1219.

[50] Albertine J. Oldehinkel, Johan Ormel, Nienke M. Bosch, Esther M. C. Bouma, Arie M. Van Roon, Judith G. M. Rosmalen, and Harriëtte Riese. 2011. Stressed out? Associations between perceived and physiological stress responses in adolescents The TRAILS study. Psychophysiology 48, 4 (2011), 441-452.

[51] Pablo E. Paredes, Nur Al-Huda Hamdan, Dav Clark, Carrie Cai, Wendy Ju, and James A. Landay. 2017. Evaluating In-Car Movements in the Design of Mindful Commute Interventions: Exploratory Study. Journal of Medical Internet Research 19, 12, Article e372 (2017), 19 pages

[52] Pablo E. Paredes, Yijun Zhou, Nur Al-Huda Hamdan, Stephanie Balters, Elizabeth Murnane, Wendy Ju, and James A. Landay. 2018. Just Breathe: In-Car Interventions for Guided Slow Breathing. Proceedings of the ACM on Interactive, Mobile, Wearable and Ubiquitous Technologies 2, 1, Article 28 (2018), 23 pages.

[53] Fabien Ringeval, Björn Schuller, Michel Valstar, Jonathan Gratch, Roddy Cowie Stefan Scherer, Sharon Mozgai, Nicholas Cummins, Maximilian Schmitt, and Maja Pantic. 2017. AVEC 2017: Real-life Depression, and Affect Recognition Workshop and Challenge. In Proceedings of the 7th Annual Workshop on Audio/Visual Emotion Challenge. 3133953, 3-9.

[54] James A. Russell. 1980. A Circumplex Model of Affect. Journal of Personality and Social Psychology 39, 6 (1980), 1161-1178.

[55] Carol D. Ryff. 2014. Psychological Well-Being Revisited: Advances in the Science and Practice of Eudaimonia. Psychotherapy and Psychosomatics 83, 1 (2014),
$10-28$

[56] Pedro Sanches, Axel Janson, Pavel Karpashevich, Camille Nadal, Chengcheng Qu, Claudia Daudén Roquet, Muhammad Umair, Charles Windlin, Gavin Doherty, Kristina Höök, and Corina Sas. 2019. HCI and Affective Health: Taking stock of a decade of studies and charting future research directions. In Proceedings of the Conference on Human Factors in Computing Systems (CHI). Article 245, 17 pages.

57] E. Glenn Schellenberg, Isabelle Peretz, and Sandrine Vieillard. 2008. Liking for happy- and sad-sounding music: Effects of exposure. Cognition and Emotion 22, 2 (2008), 218-237.

[58] Jessica Schroeder, Chelsey Wilkes, Kael Rowan, Arturo Toledo, Ann Paradiso, Mary Czerwinski, Gloria Mark, and Marsha M. Linehan. [n.d.]. Pocket skills: A conversational mobile web app to support dialectical behavioral therapy. In Proceedings of the Conference on Human Factors in Computing Systems (CHI). Article 398, 15 pages.

[59] Emery Schubert. 2004. Modeling Perceived Emotion With Continuous Musica Features. Music Perception: An Interdisciplinary fournal 21, 4 (2004), 561-585.

[60] Rob Semmens, Nikolas Martelaro, Pushyami Kaveti, Simon Stent, and Wendy Ju. 2019. Is Now A Good Time? An Empirical Study of Vehicle-Driver Communication Timing. In Proceedings of the Conference on Human Factors in Computing Systems (CHI). Article 637, 12 pages.

[61] John A. Sloboda, Susan A. O’Neill, and Antonia Ivaldi. 2001. Functions of Music in Everyday Life: An Exploratory Study Using the Experience Sampling Method. Musicae Scientiae 5, 1 (2001), 9-32.

[62] Statistisches Bundesamt. 2017. Ergebnisse des Mikrozensus 2016. Report Report EVAS-Nr. 12211. Statistisches Bundesamt. https://www.destatis.de/DE/Themen/ Arbeit/Arbeitsmarkt/Erwerbstaetigkeit/Tabellen/pendler1.html?nn=206552

[63] Maartje ter Hoeve, Robert Sim, Elnaz Nouri, Adam Fourney, Maarten de Rijke, and Ryen W. White. 2020. Conversations with Documents: An Exploration of Document-Centered Assistance. In Proceedings of the Conference on Human Information Interaction and Retrieval. 43-52.

[64] U.S. Census Bureau. 2019. 2018 American Community Survey: Commuting to Work. https://data.census.gov/cedsci/table?q=S08\&d=ACS\%201Year\%20Estimates\%20Subject\%20Tables\&tid=ACSST1Y2018.S0801 Accessed: 04/14/2020.

[65] Nicholas T. Van Dam, Marieke K. van Vugt, David R. Vago, Laura Schmalzl, Clifford D. Saron, Andrew Olendzki, Ted Meissner, Sara W. Lazar, Catherine E. Kerr, and Jolie Gorchov. 2018. Mind the hype: A critical evaluation and prescriptive agenda for research on mindfulness and meditation. Perspectives on Psychological Science 13, 1 (2018), 36-61.

[66] Annelies Van Goethem and John Sloboda. 2011. The functions of music for affect regulation. Musicae Scientiae 15, 2 (2011), 208-228.

[67] Theo Vos, Christine Allen, Megha Arora, Ryan M. Barber, Zulfiqar A. Bhutta, Alexandria Brown, Austin Carter, Daniel C. Casey, Fiona J. Charlson, and Alan Z. Chen. 2016. Global, regional, and national incidence, prevalence, and years lived with disability for 310 diseases and injuries, 1990-2015: a systematic analysis for the Global Burden of Disease Study 2015. The Lancet 388, 10053 (2016), 1545-1602.

[68] David Watson, Lee A. Clark, and Auke Tellegen. 1988. Development and validation of brief measures of positive and negative affect: the PANAS scales. Fournal of Personality and Social Psychology 54, 6 (1988), 1063-1070.

[69] Kiona K. Weisel, Lukas M. Fuhrmann, Matthias Berking, Harald Baumeister, Pim Cuijpers, and David D. Ebert. 2019. Standalone smartphone apps for mental health-a systematic review and meta-analysis. npj Digital Medicine 2, 1, Article 118 (2019), 10 pages. https://doi.org/10.1038/s41746-019-0188-8

[70] Kiona K. Weisel, Anna-Carlotta Zarski, Thomas Berger, Tobias Krieger, Michael P. Schaub, Christian T. Moser, Matthias Berking, Michelle Dey, Cristina Botella, Rosa Baños, Rocio Herrero, Ernestina Etchemendy, Heleen Riper, Pim Cuijpers, Felix Bolinski, Annet Kleiboer, Dennis Görlich, Jennifer Beecham, Corinna Jacobi, and David D. Ebert. 2019. Efficacy and cost-effectiveness of guided and unguided internet- and mobile-based indicated transdiagnostic prevention of depression and anxiety (ICare Prevent): A three-armed randomized controlled trial in four European countries. Internet Interventions 16 (2019), 52-64.

[71] Alex C. Williams, Harmanpreet Kaur, Gloria Mark, Anne Loomis Thompson, Shamsi T Iqbal, and Jaime Teevan. 2018. Supporting workplace detachment and reattachment with conversational intelligence. In Proceedings of the Conference on Human Factors in Computing Systems (CHI). Article 88, 13 pages.

[72] World Health Organization. 1946. Constitution of the World Health Organization. World Health Organization, New York, NY, USA.

[73] World Health Organization. 2017. Depression and Other Common Mental Disorders: Global Health Estimates. Report WHO/MSD/MER/2017.2. World Health Organization. https://www.who.int/mental_health/management/depression/ prevalence_global_health_estimates/en/ Accessed: 05/03/2020. 